\title{
Avaliação da Formação e Trajetória Profissional na Perspectiva de Egressos de um Curso de Psicologia
}

Evaluating psychology majors' program and career trajectories from alumni perspectives

Marucia Patta Bardagi Universidade Federal do Rio Grande do Sul / Ulbra Santa Maria - RS

Lisiane Bizarro Ana Maria Jung de Andrade Alyane Audibert Maria Célia Pacheco Lassance Universidade Federal do Rio Grande do Sul

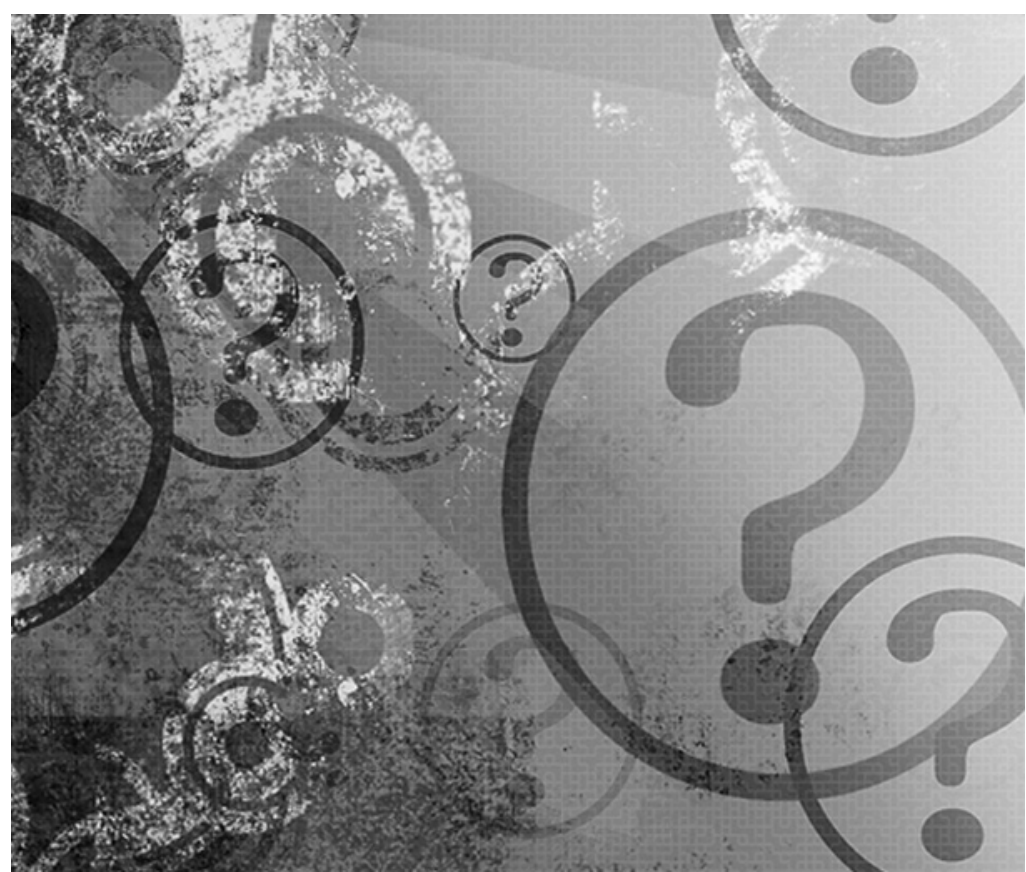


Resumo: Este estudo buscou conhecer a formação e as trajetórias de trabalho dos novos profissionais de Psicologia formados pela UFRGS. Um questionário semi-estruturado com 18 questões sobre a atuação profissional, avaliação da formação recebida e sugestões para mudanças curriculares foi enviado via internet ou correio a 143 egressos formados entre 1997 e 2004. Destes, 79 responderam (78,4\% mulheres). Os resultados indicaram que $88 \%$ dos egressos atuam como psicólogos, a maioria $(41,7 \%)$ teve a atividade clínica como primeira inserção e $88,6 \%$ buscaram formação complementar. Em relação ao curso, apontam a Psicologia clínica como a área mais privilegiada durante a graduação (29,4\%). A criação de disciplinas inexistentes e maior diversidade teórica foram as principais sugestões de mudança. Esses resultados apontam um perfil profissional ainda tradicional, um panorama favorável de inserção profissional e sugestões de mudança que vão ao encontro das novas diretrizes curriculares para os cursos de Psicologia.

Palavras-chave: Reforma curricular. Egressos. Formação profissional. Cursos de Psicologia.

\begin{abstract}
The objective of this work was to gather knowledge on the career formation and trajectories of psychologists who graduated in the Universidade Federal do Rio Grande do Sul. A semi-structured 18-topic questionnaire on professional activity, evaluation of undergraduate course and suggestions to improve curriculum was sent by e-mail or postal mail to 143 alumni, graduated between 1997 and 2004. 79 alumni (78,4\% women) replied. Results indicated that 88\% of the alumni work as psychologists, most of them had a first job as clinical psychologists $(41,7 \%)$ and $88,6 \%$ sought further academic or professional courses. Regarding the undergraduation course, 29,4\% indicated that Clinical Psychology was more privileged during the course. The main suggestions for a new curriculum were the creation of non-existent disciplines and greater theoretical diversity. These results show that the professional profile of these psychologists tend to be orthodox, have a favorable professional perspective and the suggestions meet the recent curriculum directives for the Psychology courses.
\end{abstract}

Keywords: Curriculum directives. Alumni. Profissional education. Psychology courses.

Existem diversos estudos sobre a formação e a prática profissional do psicólogo no Brasil, feitos a partir de uma perspectiva regional e que buscam avaliar os contextos específicos de formação e trabalho (Campos, Largura, \& Jankovic, 1999; Coelho \& Maciel, 2002; Conselho Regional de Psicologia / 6 a Região [CRP/06], 1995; Japur \& Osório, 1998; Yamamoto, Siqueira, \& Oliveira, 1997, entre outros), mas poucos são os estudos de escopo nacional sobre o tema (Cruces, 2006). A partir da lacuna de informações sobre a situação particular dos psicólogos no Rio Grande do Sul, este estudo de levantamento buscou conhecer a formação e as trajetórias de trabalho dos novos profissionais de Psicologia da UFRGS formados entre os anos de $1997 \mathrm{e}$
2004. Pretendeu-se, ainda, investigar também se há mudanças na inserção profissional em relação aos modelos tradicionais e em relação à realidade de outras Regiões. Assim, tem-se a intenção de contribuir com as discussões acerca da realidade profissional da Psicologia e fornecer mais subsídios às propostas de mudança curricular dos cursos de Psicologia em andamento no País.

De acordo com o relatório da Conferência Mundial sobre o Ensino Superior (Organização das Nações Unidas para a Educação, a Ciência e a Cultura [UNESCO], 1999), os cenários atual e futuro do trabalho incluem: a aceleração da modificação do perfil do emprego e das competências exigidas em 
praticamente todas as profissões, uma demanda crescente de conhecimentos gerais em informática e competências de ponta nas novas tecnologias da informação e da comunicação e a multiplicação dos papéis profissionais que exigem alto nível de conhecimento nas mais diferentes áreas. A Universidade teria o papel de fornecer, além de espaços de discussão sobre essas transformações, a oportunidade do desenvolvimento de competências relativas às mesmas. No caso brasileiro, há muito tempo, a Universidade falha em estabelecer uma interlocução sistêmica entre formação, mundo do trabalho e desenvolvimento da sociedade (Pazeto, 2005).

Nos últimos anos, nos diferentes cursos e áreas de formação, as discussões sobre reforma curricular têm emergido com crescente intensidade. A formação em Psicologia, especificamente, tem sido objeto

Na área da saúde, por exemplo, percebe-se que a maioria dos profissionais não está preparada para o trabalho fora dos contextos tradicionais. de estudos e debates, e os cursos superiores têm discutido e implementado propostas de reforma curricular para a área no Brasil. Essas discussões se iniciaram com a crítica ao Currículo Mínimo de 1962, culminando com a idéia de que a formação deve ser geral, plural, ética e científica (Gomes, 2000). Em 2001, o Parecer do Conselho Nacional de Educação/Câmara de Educação Superior no 1314 (Ministério da Educação [MEC], 2001) aprovou as novas diretrizes para os cursos de Psicologia e substituiu o "currículo baseado em enunciados de disciplinas e conteúdos programáticos por diretrizes baseadas em competências e habilidades profissionais". A Resolução n ${ }^{\circ} 8$ do Conselho Nacional de Educação/Câmara de Educação Superior, de 7 de maio de 2004 (MEC, 2004), instituiu as Diretrizes Curriculares Nacionais para os cursos de graduação em Psicologia no País. Diferente do Currículo Mínimo, as Diretrizes constituem orientações gerais, e não exigências de conteúdos.
De acordo com Gomes (2000), se, por um lado, as Diretrizes Curriculares para os cursos de Psicologia, enquanto projeto de mudança, são uma ameaça às estruturas e feudos de muitos cursos, departamentos e universidades, em contrapartida elas oferecem uma oportunidade de reforma e integração, garantindo simultaneamente a homogeneidade e a diversidade de formação. A formação generalista e a ampliação das experiências práticas durante o curso superior seriam alternativas para atender à exigência de um perfil multiprofissional e proporcionar a maturidade pessoal e a identidade profissional necessárias para agir em situações de imprevisibilidade (Gondim, 2002). Ao longo desse processo, tornou-se necessário avaliar a qualidade da formação recebida e estabelecer novos padrões para o ensino da Psicologia que se integrem às necessidades atuais decorrentes das transformações da profissão nos últimos anos.

Na área da saúde, por exemplo, percebese que a maioria dos profissionais não está preparada para o trabalho fora dos contextos tradicionais. No caso da Psicologia, a formação concentrava-se nos contextos da clínica, da escola e das organizações, e os psicólogos, de forma geral, tendem a generalizar o modelo clínico hegemônico para outras áreas e contextos de atuação, o que gera dificuldades (Dimenstein, 1998). Ao mesmo tempo em que ocorre, nos cursos de graduação, a supervalorização do modelo clínico e do perfil do psicólogo como um profissional liberal, outras formas de inserção profissional cresceram nos últimos tempos, como a atuação institucionalizada em unidades de saúde, por exemplo. Dessa forma, é consenso que os cursos de Psicologia precisam oferecer uma formação mais plural, que contemple conhecimentos sobre as áreas emergentes em Psicologia e oportunize a atuação em contextos diferenciados de intervenção, 
pesquisa ou docência (Campos et al., 1999; Carvalho, 2000; Nascimento, Manzini, \& Bocco, 2006; Noronha, 2003).

Ainda que as possibilidades de atuação do psicólogo tenham se ampliado, a Psicologia clínica continua sendo vista, tanto pelas pessoas leigas quanto pelos próprios profissionais ou recém-formados, como a área de atuação mais tradicional e aquela com maior inserção (CRP/06, 1995; Cruces, 2006; Japur \& Osório, 1998; Noronha, 2003; Yamamoto, Siqueira, \& Oliveira, 1997). Entretanto, ocorreu também o crescimento de uma representação mais diversificada do trabalho do psicólogo, com atuações mais expressivas em áreas como Psicologia da saúde, do esporte e jurídica, entre outras, o que ampliou os espaços de inserção desse profissional no mercado de trabalho (Cruces, 2006; Japur \& Osório, 1998; Praça \& Novaes, 2004; Yamamoto et al., 1997).

Nos últimos anos, foram realizados estudos de caracterização da profissão, com mapeamento de áreas de inserção, formação dos profissionais e mudanças de perfil ocorridas. A maioria dos estudos enfoca os contextos do Sudeste (Baptista, Amadio, Rodrigues, Santos, \& Palludetti, 2004; Campos et al., 1999; CRP/06, 1995; Ferrari, 2004; Japur, 1996; Japur \& Osório, 1998; Maluf, 1996; Melo-Silva \& Reis, 1997), sendo as outras Regiões sub-representadas (Coelho \& Maciel, 2002; Yamamoto et al., 1997). Esses estudos, realizados tanto com formandos quanto com alunos ainda em graduação, mostraram um panorama relativamente uniforme de trabalho, formação e imaginário social em Psicologia, além do predomínio feminino já conhecido entre os alunos e/ou profissionais (Krawulski \& Patrício, 2005; Magalhães, Straliotto, Keller, \& Gomes, 2001).

Entre os estudos com profissionais, fica evidente a ênfase na atuação em Psicologia clínica e na atividade autônoma, a maior abertura de espaço no setor público de saúde, o acúmulo de atividades, a busca por cursos de complementação da formação e ainda um índice alto de absorção dos profissionais participantes pelo mercado, entre 75 e 90\%, maior entre os egressos das universidades públicas (Japur \& Osório, 1998).

Os estudos com alunos de Psicologia apontaram certa idealização do papel do psicólogo, como um profissional que "ajuda as pessoas" (modelo assistencialista), que tem um perfil profissional especial para essa atividade, na qual a remuneração tem pouca importância (Magalhães et al., 2001). Além disso, os alunos têm a intenção de realizar cursos de aperfeiçoamento e de atuar na área clínica, mesmo que não unicamente. Os aspectos positivos dos cursos citados tanto por psicólogos quanto por alunos são a amplitude de oportunidades de estágios, a formação generalista e o contato com professores; os aspectos negativos mais relevantes são as poucas oportunidades práticas, a falta de estrutura e a falta de adequação da formação ao mercado de trabalho. Embora esses resultados de pesquisa sejam consistentes na descrição de um perfil profissional de Psicologia, generalizações são indevidas, uma vez que os estudos se concentram em uma Região do País e podem refletir uma realidade circunscrita (Japur \& Osório, 1998; Maluf, 1996). Dessa forma, há necessidade de diversificação dos estudos com alunos e egressos a fim de ampliar a visão sobre a profissão e especificar vicissitudes relativas à atuação e formação nos diferentes contextos socio-culturais do País.

No Rio Grande do Sul, especificamente, o curso de Psicologia da UFRGS teve início em 1973, no Instituto de Filosofia e Ciências Humanas, obtendo reconhecimento em 28/06/1979. Em 1996, o conselho universitário aprovou a criação do Instituto de Psicologia, composto 
A realização de pesquisas com egressos, além de auxiliar as reflexões atuais sobre reforma curricular, pode contribuir ainda para a formação de uma visão mais realista sobre a Psicologia da parte dos alunos de graduação e da comunidade em geral. por três departamentos: Psicologia do Desenvolvimento e da Personalidade, Psicologia Social e Institucional, Psicanálise e Psicopatologia (Gauer \& Gomes, 2002). Atualmente, o curso conta com um corpo docente de 37 professores, sendo que 31 têm a titulação de Doutor, além de dois cursos de pós-graduação strictu senso. Até a reforma curricular, em 2006, o currículo estabelecia uma carga horária fixada em 4155 horas, distribuídas em 10 semestres, tendo o aluno que completar 250 créditos obrigatórios, incluindo quatro estágios curriculares obrigatórios (psicopatologia, Psicologia escolar, Psicologia do trabalho e Psicologia clínica) e 27 créditos eletivos. No período compreendido pelo presente estudo (1997-2004), o curso formou 238 alunos. A ausência de estudos com egressos da Universidade não permite uma comparação entre os perfis profissionais e de formação do aluno da UFRGS com aqueles de outras universidades e Regiões já descritos acima. Dessa forma, para reunir informações que servissem como auxílio às discussões da reforma curricular e permitir uma comparação com resultados de outros estudos, este levantamento buscou identificar as percepções do aluno egresso sobre a própria formação e sua trajetória profissional no mercado de trabalho. A realização de pesquisas com egressos, além de auxiliar as reflexões atuais sobre reforma curricular, pode contribuir ainda para a formação de uma visão mais realista sobre a Psicologia da parte dos alunos de graduação e da comunidade em geral.

\section{Método}

Participantes: participaram deste estudo 79 psicólogos (78,4\% mulheres) egressos do curso de Psicologia da Universidade Federal do Rio Grande do Sul, formados entre 1997 e o primeiro semestre de 2004. Esse número representa 33,2\% do total de egressos. Dos 238 formados no período, foram contatados, ao todo, 143 egressos, obtendo-se uma taxa de adesão de 55,2\% ao estudo. A Tabela 1 apresenta uma descrição do número de participantes por ano.

Tabela 1. Número de participantes em relação ao total de egressos do período.

\begin{tabular}{lccccccccc}
\hline & 1997 & 1998 & 1999 & 2000 & 2001 & 2002 & 2003 & $\begin{array}{c}2004 / \\
1\end{array}$ & $\begin{array}{c}\text { Participantes/ } \\
\text { total }\end{array}$ \\
\hline Homens & $1 / 5$ & $5 / 13$ & $2 / 6$ & $2 / 6$ & $1 / 6$ & $2 / 8$ & $2 / 7$ & $2 / 2$ & $17 / 53$ \\
Mulheres & $8 / 25$ & $11 / 21$ & $13 / 29$ & $9 / 24$ & $5 / 28$ & $9 / 33$ & $7 / 23$ & $0 / 2$ & $62 / 85$ \\
Participantes/total & $9 / 30$ & $16 / 34$ & $15 / 35$ & $11 / 30$ & $6 / 34$ & $11 / 41$ & $9 / 30$ & $2 / 4$ & $79 / 238$ \\
\hline
\end{tabular}

Instrumento e procedimentos: um questionário semi-estruturado com 18 questões foi elaborado para identificar a trajetória profissional do egresso e suas impressões sobre a formação acadêmica. Entre as informações solicitadas, estavam as atividades atuais dos profissionais, a abordagem teórica com a qual eles trabalham, as atividades de formação complementar realizadas e a avaliação do curso realizado, que incluem as sugestões para modificação curricular e a percepção de quais foram as disciplinas mais importantes, entre outras. O modelo do questionário encontra-se no Anexo A. 
Os nomes e contatos dos possíveis participantes da pesquisa foram obtidos através de indicações de diversos setores do Instituto de Psicologia da UFRGS, além de consultas a professores, alunos e outros egressos. A partir dessas indicações, os participantes foram contatados por telefonemas ou mensagens de e-mail que explicavam os objetivos e a metodologia da pesquisa e solicitavam a autorização para envio do questionário como anexo a uma mensagem eletrônica. Os participantes que não tinham acesso a correio eletrônico receberam o instrumento impresso pelo correio, com envelope para retorno selado endereçado a uma das pesquisadoras no Instituto de Psicologia. Para garantir o sigilo e a confidencialidade dos dados, os questionários foram todos codificados e impressos sem a identificação do participante. Os participantes receberam a devolução dos resultados da pesquisa, por correio eletrônico, com os trabalhos científicos já produzidos e apresentados sobre o estudo em eventos.

\section{Resultados}

Os questionários foram submetidos à análise de conteúdo (Bardin, 1977), com o levantamento de percentuais para os dados quantitativos e a extração das categorias emergentes para cada questão aberta, em um critério de consenso entre quatro juízes. Foi feita ainda uma contagem das respostas em cada categoria e obtidas freqüências. Os resultados estão organizados em duas sessões: a trajetória profissional do egresso e a avaliação da formação recebida.

1) Trajetória profissional:

Os resultados revelam que $88 \%$ dos egressos atuam como psicólogos, sendo que $92 \%$ deles são remunerados. A maioria, conforme observado na Tabela 2, teve a atividade clínica (33,8\%) ou a pós-graduação (25\%) como primeira inserção, mantendo-se estas como as atividades atuais mais citadas. A categoria "outras" tem um percentual elevado por englobar um conjunto de 13 atividades, sendo as mais citadas: prestar consultoria, ministrar curso/palestras, Psicologia da saúde/hospitalar e atividades práticas de extensão/residência/especialização. 43\% dos profissionais exercem, ainda, mais de uma atividade na área.

Tabela 2. Trajetória profissional dos alunos egressos da UFRGS.

\begin{tabular}{lcc}
\hline & 1ạ inserção & Atividades atuais \\
\hline Clínica / consultório particular & $35,8 \%$ & $26,4 \%$ \\
Mestrado / doutorado / pesquisa & $25 \%$ & $21,6 \%$ \\
Não relacionadas a psi & $13 \%$ & $13,6 \%$ \\
Organizacional & $10,8 \%$ & $8,8 \%$ \\
Concurso & - & $8,8 \%$ \\
Docência & - & $6,4 \%$ \\
Outras & $15,2 \%$ & $24,8 \%$ \\
\hline
\end{tabular}


A psicanálise (42\%) e a Psicologia social/institucional (15\%) foram as linhas teóricas mais citadas entre 10 abordagens referidas como base para a prática profissional. 7\% dos egressos afirmaram não adotar uma linha teórica específica para a atividade que exercem.

Durante a graduação, muitos alunos buscaram diversas atividades extracurriculares, sendo as principais a realização de cursos/grupos de estudo (28,1\%), iniciação científica $(26,5 \%)$ e participação em encontros científicos em geral (18\%); por outro lado, após a graduação, as atividades de complemento concentraram-se em cursos de especialização/formação (54\%) e mestrado (21\%). Do total de participantes, 88,6\% realizaram algum tipo de atividade de formação complementar após a graduação. A Tabela 3 apresenta os percentuais de atividades realizadas pelos alunos durante e depois da graduação.

Tabela 3. Atividades realizadas pelos egressos durante e após a graduação.

Atividades extracurriculares importantes
Atividades de complemento à formação

\begin{tabular}{lcc}
\hline Não fez & $2,7 \%$ & $4,9 \%$ \\
Pesquisa / iniciação científica & $28,1 \%$ & - \\
Cursos / especialização / extensão & $21 \%$ & $54,2 \%$ \\
Mestrado / doutorado & - & $21,1 \%$ \\
Encontros científicos em geral & $18,2 \%$ & $5,6 \%$ \\
Supervisões & - & $2,1 \%$ \\
Estágios & $11 \%$ & $1,4 \%$ \\
Grupos de estudo / estudo individual & $7,2 \%$ & $7 \%$ \\
Outros & $11,6 \%$ & $2,1 \%$ \\
\hline
\end{tabular}

2) Formação recebida:

Do total de participantes, $48 \%$ sentiam-se preparados para atuar ao final do curso, enquanto a maioria se declarou não preparada ou "mais ou menos" preparada. Quanto aos aspectos positivos da formação recebida, as características específicas do curso $(23,7 \%)$, os estágios (23,7\%) e a qualidade dos professores $(12,8 \%)$ foram os tópicos mais citados pelos participantes. Como características específicas do curso, foram citados aspectos como o incentivo à pesquisa, o desenvolvimento de um espírito crítico e de autonomia durante a graduação, o desenvolvimento de uma postura ética e profissional e o fornecimento de uma formação generalista, entre outros.

Muitos egressos consideraram que o curso privilegia algumas áreas em detrimento de outras. Os participantes relataram que a Psicologia clínica foi a área mais privilegiada durante a graduação $(29,4 \%)$, e que a Psicologia organizacional foi a área que recebeu menor atenção (25\%). A Tabela 4 apresenta as respostas mais freqüentes para essa questão. 
Tabela 4. Áreas mais e menos privilegiadas do curso na opinião dos egressos.

\begin{tabular}{cccc}
\hline Áreas privilegiadas & \multicolumn{3}{c}{ Áreas menos privilegiadas } \\
\hline Nenhuma & $14,3 \%$ & Não sabe / Não respondeu & $3,2 \%$ \\
Psicologia clínica & $37,8 \%$ & Organizacional & $30,7 \%$ \\
Pesquisa/Academia & $15,1 \%$ & Psicologia da saúde & $6,4 \%$ \\
Psicologia social & $12,6 \%$ & Psicologia do esporte & $5,7 \%$ \\
Teoria psicanalítica & $8,4 \%$ & Teoria junguiana & $3,8 \%$ \\
\hline
\end{tabular}

O período considerado mais difícil do curso foi o período intermediário $(44,2 \%)$, que compreende do 5o ao 7ํㅗ semestre, quando há aumento da carga horária e o início dos estágios. Um número significativo de sugestões de mudanças estruturais e curriculares foi apresentado pelos participantes. A Tabela 5 apresenta as mais freqüentes.

Tabela 5. Sugestões sobre mudanças curriculares para o Curso de Psicologia da UFRGS.

\section{Criação de disciplinas inexistentes}

(ex.: habilidades gerais, Filosofia, políticas públicas, organizacional,

cognitivo-comportamental, etc)

\section{Mudanças na estrutura do curso}

(ex.: maior integração teoria e prática, mais atividades práticas e

contato com o mercado, integração entre departamentos, etc.)

\section{Maior diversidade teórica}

(ex.: menos psicanálise, aproximação com outros cursos, equilíbrio entre teorias, etc.)

\section{Aprofundar o conteúdo de disciplinas existentes}

(ex.: mais semestres, mais conteúdos, mudanças

na súmula, etc)

\section{Modificações no quadro ou didática dos professores}

(ex.: maior comprometimento com a graduação, mudanças de didática, atuação profissional fora da universidade)

\section{Mudanças nos estágios}

(ex.: menor quantidade, maior possibilidade de escolha, outras 


\section{Discussão}

A partir dos resultados deste estudo, concluise que, entre os egressos do curso de Psicologia da UFRGS, a Psicologia clínica é predominante, tanto como início de atividade profissional quanto como projeto de carreira, o que corrobora a literatura (CRP/06, 1995; Cruces, 2006; Noronha, 2003; Yamamoto et al., 1997). Embora o campo de atuação da Psicologia tenha se diversificado nos últimos anos, os psicólogos formados na UFRGS parecem preferir, ainda, formas de inserção já consolidadas e mais próximas do imaginário social da profissão. Provavelmente, essa preferência é influenciada também pela formação oferecida pelo curso na época, que ainda adotava o modelo do Currículo Mínimo de 1962, que privilegia a Psicologia clínica, na opinião dos egressos. No entanto, mesmo que em número reduzido, o estudo mostra um início de ampliação da prática profissional, indicando atividades atuais em áreas aplicadas emergentes, como, por exemplo, Psicologia jurídica, hospitalar, comunitária, publicitária e do esporte. Assim como em outros estudos, reitera-se a necessidade de se ampliar o escopo da profissão para além da área clínica, trazendo à tona novas formas de inserção profissional (Dimenstein, 1998; Noronha, 2003; Pazeto, 2005; Yamamoto et al., 2003).

A inserção dos egressos em áreas aplicadas emergentes não reflete diretamente a formação teórica recebida no curso. Isso fica evidenciado pelas sugestões de mudanças curriculares, como novas disciplinas e maior diversidade teórica. Sendo assim, a inserção em áreas emergentes possivelmente é um produto da formação buscada pela grande maioria após a graduação. Cursos de formação são uma trajetória natural na continuidade dos estudos (Cruces, 2006). Entretanto, concomitantemente, observou-se uma redução na participação em encontros científicos, que são oportunidades de atualização e troca de experiências das quais os egressos participavam quando eram estudantes. Parece que a participação em eventos científicos foi estimulada durante o curso, mas, após a graduação, os profissionais focalizam seus investimentos mais em cursos de formação do que nesse tipo de evento. A ciência e a profissão parecem estar dissociadas quando o psicólogo entra no mercado de trabalho.

Em geral, os egressos relataram satisfação com a formação recebida e apresentaram boa inserção no mercado. A maioria atua como psicólogo, embora nem sempre com remuneração. Um ponto a ser salientado é o alto índice de inserção profissional, especialmente considerando o pouco tempo de formação de muitos dos participantes. Isso pode indicar, entre outros motivos, a qualidade dos profissionais e da formação recebida, as boas possibilidades do mercado e, como aponta o CRP/06 (1995), a grande absorção de alunos da universidade pública pelo mercado de trabalho. Também é relevante considerar aqui não só a qualidade dos profissionais formados mas também as demandas de um contexto conservador, que privilegia uma formação tradicional.

É importante considerar os aspectos apontados como positivos do curso no processo de reforma curricular, mas devem ser levadas em conta as sugestões de mudança, uma vez que a percepção da necessidade de 
diversificação teórica e de flexibilização do currículo vem ao encontro das novas diretrizes para os cursos de Psicologia, que, espera-se, representem mudanças que possam contemplar as novas necessidades acadêmicas e profissionais da área. O incentivo à pesquisa, o desenvolvimento da autonomia e do espírito crítico e a qualidade dos professores são aspectos positivos relacionados ao contexto da universidade pública e ao ambiente de pesquisa oferecido pelos cursos de pós-graduação do Instituto de Psicologia.

Ao mesmo tempo em que houve a sugestão de redução no número de estágios curriculares, a experiência dos estágios foi considerada um aspecto positivo na formação. É possível que os estágios tenham cumprido o papel de integrar prática e teoria para muitos, ainda que tenha havido a sugestão de incrementar essa integração nas disciplinas. A diversidade dos estágios curriculares (psicopatologia, Psicologia clínica, Psicologia escolar e Psicologia do trabalho) faz parte do objetivo de oferecer uma formação generalista, e essas múltiplas experiências no campo profissional potencializam a possibilidade de uma inserção bem sucedida.

No entanto, algumas considerações metodológicas são importantes. Como o número total de participantes é relativamente pequeno para representar a realidade do aluno egresso da Psicologia da UFRGS, há necessidade de um levantamento mais extensivo das condições de trabalho e da inserção dos formados pela instituição. Observou-se, por exemplo, que 25\% dos participantes tiveram como primeira inserção a pós-graduação, o que parece superestimado e certamente reflete a facilidade de contactar esses egressos que permanecem no meio universitário. Além disso, a maioria dos participantes está em início de carreira profissional e possui, em conseqüência, trajetórias iniciais de inserção. É importante também avaliar, em novos estudos, a realidade ocupacional de alunos formados há mais tempo e acompanhar, ainda, a partir de agora, as trajetórias de inserção dos alunos formados dentro de outra perspectiva curricular diferente daquela do Currículo Mínimo de 1962 a fim de avaliar se a proposta de mudança no processo de formação se reflete em diferenças na atividade profissional do egresso. Outra consideração é que grande número de pessoas que responderam estão satisfeitas com a profissão e empregadas, o que pode trazer um viés ao estudo, pois aqueles alunos que estão fora do mercado ou estão descontentes com a profissão podem ter se recusado a participar, realidade observada também em outras pesquisas (Yamamoto et al., 2003). Para fins de uma avaliação institucional, justamente esses alunos poderiam fornecer uma contribuição maior, com outros olhares sobre a formação e a realidade profissional vivenciada.

Conclui-se, no entanto, que, como levantamento sobre as trajetórias de carreira e as percepções sobre a formação recebida, este estudo pode fornecer informações relevantes, tanto para o conhecimento da realidade profissional quanto para as discussões de mudanças curriculares. Seria interessante que houvesse um processo sistematizado de avaliação do ex-aluno em todas as instituições de ensino superior como ferramenta de retroalimentação privilegiada. 


\section{Marucia Patta Bardagi}

Doutora em Psicologia - UFRGS/Ulbra Santa Maria-RS

Lisiane Bizarro

Doutora em Psicologia - UFRGS

Ana Maria Jung de Andrade

Psicóloga - UFRGS

Alyane Audibert

Graduanda de Psicologia - UFRGS

Maria Célia Pacheco Lassance *

Mestre em Educação - UFRGS

* Endereço para correspondência: Universidade Federal do Rio Grande do Sul, Instituto de Psicologia, Rua Ramiro Barcelos, 2600, Cep: 90035-003, Porto Alegre-RS.

E-mail: maria.lassance@ufrgs.br 


\section{Referências}

Baptista, M. N., Amadio, A., Rodrigues, E. C., Santos, K. M., \& Palludetti, S. A. T. (2004). Avaliação dos hábitos, conhecimentos e expectativas de alunos de um curso de Psicologia. Psicologia Escolar e Educacional, 8(2), 207-217.

Bardin, L. (1997). Análise de conteúdo. Lisboa: Edições 70.

Campos, L. F., Largura, W. A. N., \& Jankovic, A. L. (1999). Efeito da graduação em psicologia nas escolhas profissionais de seus estudantes. Psico, 30(2), 29-44.

Carvalho, A. M. (2000). A formação em psicologia e as diretrizes curriculares: perspectivas e desafios [Resumo]. In Anais, $1^{\circ}$ Encontro Sul-Brasileiro de Psicologia (pp. 72-75). Curitiba.

Coelho, L. D., \& Maciel, D. A. (2002). A perspectiva de formandos acerca do curso de graduação em psicologia da UNB: um estudo exploratório. Arquivos Brasileiros de Psicologia, 54(2), 144-157.

Conselho Regional de Psicologia - 6a Região. (1995). Psicologia: formação, atuação profissional e mercado de trabalho - São Paulo, Mato Grosso e Mato Grosso do Sul. Estatísticas 1995. São Paulo: Autor.

Cruces, A. (2006). Egressos de cursos de psicologia: preferências, especializações, oportunidades de trabalho e atuação na área educacional. Tese de Doutorado, Programa de Pós-Graduação em Psicologia Escolar, Instituto de Psicologia, Universidade de São Paulo, São Paulo.

Dimenstein, M. D. B. (1998). O psicólogo nas unidades básicas de saúde: desafios para a formação e atuação profissionais. Estudos de Psicologia, Natal, 3(1), 53-81.

Ferrari, I. F. (2004). Os cursos de psicologia de Minas Gerais e a presença da psicanálise na disciplina psicopatologia. Revista Mal-Estar na Subjetividade, 4(2), 372-391.

Gauer G., \& Gomes, W. B. (2002). O curso da reforma: ensino de psicologia na Universidade Federal do Rio Grande do Sul (1971-1979). Psicologia: Reflexão e Crítica, 15(3), 497-513.

Gomes, W. B. (2000). Diretrizes curriculares e perspectivas de mudanças na formação em psicologia [Resumos]. In Anais, $1^{\circ}$ Encontro Sul-Brasileiro de Psicologia (pp. 81-82). Curitiba.

Gondim, S. M. G. (2002). Perfil profissional e mercado de trabalho: relação com a formação acadêmica pela perspectiva de estudantes universitários. Estudos de Psicologia, Natal, 7(2), 299-309.

Japur, M. (1996). Formação em psicologia: análise dos aspectos estruturais de um curso de graduação. Paidéia, 10/11, 131148.

Japur, M., \& Osório, F. L. (1998). Avaliação de um curso de psicologia em relação à área de inserção profissional. Psico, 29(1), 7-32.
Krawulski, E., \& Patrício, Z. M. (2005). Por que pessoas escolhem a psicologia como profissão?. In M. C. P. Lassance, A. C. Paradiso, M. P. Bardagi, M. Sparta, \& S. L. Frischenbruder (Orgs.), Intervenção e compromisso social: orientação profissional teoria e técnica (Vol. 2, pp. 323-336). São Paulo: Vetor.

Magalhães, M., Straliotto, M., Keller, M., \& Gomes, W. B. (2001). Eu quero ajudar as pessoas. Psicologia: Ciência e Profissão, 21, 10-27.

Maluf, M. R. (1996). Psicólogo brasileiro: formação, problemas e perspectivas. In E. M. Bomfim (Org.), Formações em psicologia: pós-graduação e graduação (Coletâneas da ANPEPP, pp. 7186). Belo Horizonte: ANPEPP/UFMG.

Melo-Silva, L. L., \& Reis, V. A. B. (1997). A identidade profissional em estudantes do curso de Psicologia: intervenção através da técnica de grupo operativo. In Anais, III Simpósio Brasileiro de Orientadores Profissionais (pp. 57-65). Canoas, RS.

Ministério da Educação. (2001). Parecer CNE/CES n. ${ }^{\circ}$ 1.314, de 7 de novembro de 2001. Recuperado em 2001, de www. portal.mec.gov.br/cne/index.php?option $=$ content $\&$ task $=$ vie w\&id $=98 \&$ Itemid $=227$

Ministério da Educação. (2004). Resolução nº 8, de 7 de maio de 2004. Resolução do Conselho Nacional de Educação/Câmara de Educação Superior. Recuperado em 2004, de www.portal. mec.gov.br/cne/arquivos/pdf/rces08_04.pdf

Nascimento, M. L., Manzini, J. M., \& Bocco, F. (2006). Reinventando as práticas psi. Psicologia \& Sociedade, 18, 15-20.

Noronha, A. P. (2003). Docentes de psicologia: formação profissional. Estudos de Psicologia, Natal, 8(1), 169-173.

Organização das Nações Unidas para a Educação, a Ciência e a Cultura. (1999). Conferência mundial sobre o ensino superior - Tendências da educação superior para o século XXI, 1998, Paris. Brasília, DF: UNESCO/CRUB.

Pazeto, A. E. (2005). Universidade, formação e mundo do trabalho: superando a visão corporativa. Ensaio. Avaliação, Políticas Públicas e Educação, 13(49), 487-496.

Praça, K. B. D., \& Novaes, H. G. V. (2004). A representação social do trabalho do psicólogo. Psicologia: Ciência e Profissão, 24, 32-47.

Yamamoto, O. H., Dantas, C. M. B., Costa, A. L. F., Alverga, A. R., Seixas, P. S., \& Oliveira, I. F. (2003). A profissão de psicólogo no Rio Grande do Norte. Interação, 7(2), 23-30.

Yamamoto, O. H., Siqueira, G. da S., \& Oliveira, S. C da C. (1997). A psicologia no Rio Grande do Norte: caracterização geral da formação acadêmica e do exercício profissional. Estudos de Psicologia, Natal, 2(1), 42-67. 